\section{La razón rentista y sus teóricos: la economía política en el siglo petrolero venezolano}

\author{
The Rentier Reason and Its Theorists. \\ The Political Economy in the Venezuelan \\ Oil Century
}

\author{
Malfred Gerig* \\ CENTRO NACIONAL DE ESTUDIOS HISTÓRICOS \\ CARACAS, VENEZUELA \\ $\triangle$ Malfredgerig7@gmail.com \\ https://orcid.org/0000-0002-8030-9176
}

\author{
Anuario Latinoamericano \\ Ciencias Políticas \\ y Relaciones Internacionales \\ vol. 9, 2020 \\ pp. $13-36$
}

DOI: 10.17951/al.2020.9.13-36

\title{
RESUMEN
}

Este artículo se plantea analizar la conexión entre la historia intelectual de la tradición de pensamiento sobre la economía política del petróleo y la historia económica de Venezuela durante el siglo petrolero venezolano. Para ello: 1) se caracteriza la razón rentista, 2) se propone una interpretación de la relación entre la historia intelectual y la historia económica de Venezuela en el siglo XX, 3) se analiza la tradición de economía política del petróleo a la luz de los cambios políticos y económicos, 4) se analiza la tradición abocada a la economía política del petróleo y su relación con el ocaso del siglo petrolero venezolano, 5) se expone una interpretación sobre la crisis en la que se encuentra la sociedad venezolana.

PALABRAS CLAVE: Venezuela, petróleo, economía política, renta del petróleo.

\section{ABSTRACT}

This article aims to analyze the connection between the intellectual history of the tradition of thought on the political economy of oil and the economic history of Venezuela during the Venezuelan oil century. To do this: 1) rentier reason is characterized, 2) an interpretation of the relationship between intellectual history and economic history in Venezuela in the $20^{\text {th }}$ century is proposed, 3) the tradition of the political economy of oil is analyzed in light of political and economic changes,

* Sociólogo por la Universidad Central de Venezuela (UCV), candidato a magister en Ciencia Política por la Universidad Simón Bolívar (USB), director ejecutivo de la consultora Laboratorio Estratégico e investigador del Centro Nacional de Estudios Históricos (CNEH). 
Dossier

América Latina:

Venezuela
4) the tradition focused on the political economy of oil and its relationship with the decline of the Venezuelan oil century are analyzed, 5) an interpretation of the crisis in Venezuelan society is presented.

KEYWORDS: Venezuela, petroleum, political economy, oil income.

\section{Introducción}

Desde 2012 Venezuela ha vivido una transformación súbita en la forma de entender los arreglos económicos, sociales y geopolíticos. El siglo petrolero venezolano, que se inicia en 1917 con la primera exportación de petróleo y tiene su punto de apogeo en 2005, cuando las exportaciones de bienes y servicios se ubicaron al 39,6 \% del Producto Interno Bruto (PIB), a partir de 2012 llegó a un momento de crisis, colapso y extinción. El objetivo de este trabajo es analizar la conexión entre la historia intelectual de la tradición de pensamiento abocada a la economía política del petróleo y la historia económica de Venezuela durante el siglo petrolero venezolano.

Las tesis que se traen a colación son: primero, que el pensamiento abocado a la economía política del petróleo en Venezuela ha estado dividido en dos vertientes. Por un lado, la política económica del petróleo se ha ocupado de legitimar el ingreso rentista. Por el otro, la economía política del petróleo se ha propuesto la recta caracterización económica del recurso natural y el tipo de ingreso que genera. Segundo, que la razón rentista ha sido la meta-estrategia que ha dominado el siglo XX en Venezuela en su inserción en la economíamundo capitalista. Tercero, que la historia económico-social y la historia intelectual del siglo petrolero venezolano han estado inseparablemente unidas, mientras que en la fase de ascenso del capitalismo rentístico ha predominado la política económica y en la fase de descenso la economía política.

Metodológicamente, el análisis adopta tanto una perspectiva histórica y global como un enfoque teórico e interpretativo para dar cuenta de la mediación dialéctica entre la emergencia de ideas sobre la economía política del petróleo, la modificación de prácticas económico-sociales y la aparición de cambios políticos, institucionales y dinámicas económicas. El trabajo abarca un marco cronológico que va desde 1917 hasta principios del siglo XXI cuando se dilucida el agotamiento del siglo petrolero venezolano.

\section{La razón rentista}

En 1913, el ministro de fomento de Venezuela, Pedro Emilio Coll, declaró en su informe ante el Congreso que "en breves días podemos contar con una nueva fuente de producción rentista que no tardará en ser la de mayor importancia. El petróleo (...) ha dejado de ser tesoro escondido en las entrañas de 
la tierra venezolana" (citado en Salas, 2013, p.74). Habían trascurrido cuatro siglos desde que Francisco de Castellanos, tesorero de Nueva Cádiz, le enviase un cargamento de petróleo al rey de España en 1539 hasta la inauguración del pozo Zumaque I con la cual se quebrara en dos la historia -tanto de la estructura económico-social como de las preguntas que obsesionan a su intelectualidad- de Venezuela.

A partir del Zumaque I se conformó un punto de partida, un fondo histórico y un mundo determinado que colocó a la economía política como su centro constitutivo y desde el cual emanaron una serie de interrogantes comunes sobre el país que en el devenir dieron forma a una tradición de pensamiento sobre la economía, la política y la sociedad. ¿Cuáles son las interrogantes comunes que fundan la tradición? En su núcleo, la economía política del petróleo, la tradición ha girado constantemente en torno a dos preguntas. En primer lugar: ¿qué tipo de riqueza surge del petróleo como mercancía? El carácter fundante de esta pregunta se explica por la relación directa que existe entre la determinación específica de la naturaleza económica de una mercancía y el tipo de rédito o ingreso que de ahí se deriva. Como se verá en lo sucesivo, esta es una discusión de primer orden en el seno de la tradición, con inestimables consecuencias para la vida económica del país, ya que las implicaciones en política económica serán diversas, dependiendo de si el rédito es caracterizado como una renta, un activo o un capital. En segundo lugar: ¿cuál es la forma correcta de invertir los réditos provenientes del petróleo con la intención de garantizar la reproducción de la riqueza? El carácter acuciante de esta otra interrogante se explica en la popularidad irreflexiva de su conocido dictum metafórico: sembrar el petróleo.

En el fondo de las respuestas dadas a la obsesión por la siembra del petróleo se encuentra lo que se denominará la razón rentista, esto es, la meta-estrategia que buscaba tanto legitimar como sacar provecho de la inserción dependiente o condición periférica de Venezuela en la economía-mundo capitalista en el marco del ciclo sistémico de acumulación estadounidense (Arrighi, 1999, p. 322) mediante la absorción de una renta internacional del petróleo. Expliquemos los cuatro ejes que soportan la razón rentista en las palabras de la tradición en distintos momentos del siglo petrolero venezolano:

1. El reclamo de la propiedad del Estado sobre el petróleo. En 1920, Gumersindo Torres reclamaba que “... nada pagan las empresas por el derecho mismo a la explotación (...) ora a los propietarios del suelo, comprándoles carísimas tierras petroleras, ora al Estado mismo, si el terreno es baldío, mediante especiales estipulaciones contractuales" (G. Torres citado en Baptista y Mommer, 1992, p. 10).

2. La maximización de los ingresos rentistas. A principios de la década de 1970 Juan Pablo Pérez Alfonzo (2011, p. 84) iba al quid del asunto al sostener que "el eje de la política petrolera de Venezuela desde que pudo formularse por sus gobiernos democráticos fue siempre la defensa de los precios del petróleo como base para maximizar el ingreso nacional que debe dejar su explotación".
La razón rentista y sus teóricos: la economía política en el siglo petrolero venezolano

Malfred Gerig 
Dossier América Latina: Venezuela
3. La posibilidad de invertir los ingresos rentistas en un proyecto nacional de desarrollo. En 1936, Arturo Uslar Pietri (2006, p. 22) expresaría sucintamente la principal obsesión de la razón rentista: "urge crear sólidamente en Venezuela una economía reproductiva y progresiva (...) es menester sacar la mayor renta de las minas para invertirla totalmente en ayudas, facilidades y estímulos a la agricultura, la cría y las industrias nacionales".

4. Centrar el debate político, luego de coincidir grosso modo en los puntos anteriores, en torno al sujeto social encargado de traducir el excedente de origen externo en desarrollo nacional.

La tradición de pensamiento abocada a la economía política del petróleo en Venezuela se encuentra dividida a lo interno por dos vertientes. Por un lado, la dedicada a la economía política del petróleo cuya materia principal empieza en la recta caracterización del petróleo como mercancía y se expande hacia una teoría de la naturaleza del rédito petrolero con sus concomitantes. Por el otro lado, la política económica del petróleo, la cual se ha ocupado solo de legitimar y construir posibles vías de inversión que permitan absorber y reproducir la riqueza petrolera sujetados en la razón rentista. De la primera emana una visión escéptica amén de reflexiva sobre el siglo petrolero venezolano, con sus certezas coladas más en la larga duración que en el acontecimiento, más en la estructura que en la política. De la segunda emana una visión sujeta a la oscilación del ciclo económico que recicla sus variantes optimistas en el auge y moralizantes en el declive. De la economía política del petróleo suele surgir una postura crítica de la razón rentista, mientras que en la política económica del petróleo siempre está a la orden del día la ponderación de una oportunidad para el sempiterno intento de sembrar el petróleo.

\section{Siglo petrolero venezolano y nacionalismo petrolero}

En su monumental El largo siglo XX Giovanni Arrighi sostuvo que la dinámica de comportamiento de la economía-mundo capitalista a escala sistémica se adaptaba a la fórmula general del capital de Marx (1975, p. 179). A partir de esta lógica se dibuja un ciclo sistémico de acumulación que alterna "épocas de expansión material (fases DM de acumulación de capital) con fases de renacimiento y expansión financieros (fases $\mathrm{MD}^{\prime}$ )”. En la fase material, la "fuerza de trabajo mercantilizada y los recursos naturales" son el vehículo de la acumulación de capital, mientras que en las fases financieras las agencias de acumulación de capital "prefieren la liquidez", virando hacia "procedimientos financieros" (Arrighi, 1999, p. 18-19).

$\mathrm{Al}$ igual que la fórmula general del capital de Marx (DMD') describe el comportamiento de los ciclos sistémicos de acumulación, también es útil para interpretar la dinámica de la economía venezolana durante el siglo petrolero venezolano, es decir, el periodo de inserción de Venezuela en la economíamundo capitalista como exportador de petróleo al servicio del ciclo sistémico 
de acumulación estadounidense caracterizado por ser intensivo en energía y medios de producción. De este modo, la fase de expansión material (DM) del siglo petrolero venezolano inicia con la primera exportación de petróleo, en 1917, y se extiende hasta la década 1973-1983. En este periodo la economía venezolana experimentó una fase de crecimiento estable motorizada por la razón rentista que inició con la inserción en la división internacional del trabajo y se consumó con la nacionalización de la industria petrolera en 1976.

En 1972, Venezuela gozaba de un 6,9 \% de renta del petróleo como porcentaje del PIB según el Banco Mundial (BM) $)^{1}$. En 1974, la renta ascendió al $34,2 \%$ del PIB para descender al 7,9 \% en 1986. El saldo en cuenta corriente, por su parte, ascendió del 0,9\% del PIB en 1970 al 22,06 \% en 1974, para descender al 14,6\% en 1978. La deuda externa acumulada como porcentaje del Ingreso Nacional Bruto (INB) pasó del 13,5 \% en 1970 al 63,6 \% en 1984. Por último, la formación bruta de capital fijo como porcentaje del PIB pasó de ser el 36,5\% en 1970 al 51,8 \% en 1978 para descender hasta el 14,1\% en 1983 (Banco Mundial, 2020). En el periodo 1972-1983 la economía venezolana experimentó una "crisis señal" del modo de acumulación que paradójicamente comienza con un aumento de los precios del petróleo. En febrero de 1983, con la introducción del control cambiario inició la fase de declinación financiera del siglo petrolero venezolano. Este periodo se caracteriza por el cambio discontinuo, las críticas a la senda de desarrollo llevada a cabo en el periodo de auge material, las turbulencias macroeconómicas y los intentos de restructuración política.

La historia económico-social y la historia intelectual del siglo petrolero venezolano han estado inseparablemente unidas. La fase de ascenso del capitalismo rentístico ha sido también la fase de ascenso del nacionalismo petrolero, la razón rentista y el predominio de la política económica del petróleo en detrimento de la economía política del petróleo. La fase de descenso del capitalismo rentístico ha presenciado la constatación de la imposibilidad de la siembra del petróleo, dando lugar al agotamiento del nacionalismo petrolero, el debilitamiento de la razón rentista y el ascenso de la economía política del petróleo.

El nacionalismo petrolero ha sido el "manual de actividad política cotidiana"2 del siglo petrolero venezolano, así como la "codificación política" de la razón rentista. Dos nacionalismos petroleros han cursado el siglo. El primer nacionalismo petrolero, que va desde 1917 hasta 1945, consuma la inserción rentista en el ciclo sistémico de acumulación estadounidense, teniendo como principal objetivo convertir a Venezuela en un país propietario de petróleo. El nacionalismo petrolero segundo, el cual se establece en 1945, se consuma en 1976 con la nacionalización de la industria petrolera y tiene como hito convertir a Venezuela

1 El Banco Mundial conceptualiza a la "la renta del petróleo" como "la diferencia entre el valor de la producción de petróleo crudo a precios mundiales y los costos totales de producción”.

2 El término tomado de Wallerstein (2014, pp. 21-22).
La razón rentista y sus teóricos: la economía política en el siglo petrolero venezolano Malfred Gerig 
Dossier
América Latina:

Venezuela

en un país productor de petróleo. Si el sustrato del nacionalismo petrolero es legitimar la propiedad del Estado sobre el petróleo como forma de sacar provecho a la condición periférica de Venezuela en la división internacional del trabajo mediante la absorción de una renta internacional del petróleo, la némesis del nacionalismo petrolero ha encontrado sus más fieles discípulos en las corporaciones petroleras globales y la burguesía venezolana para quienes la propiedad estatal ejerce como causa de la imposibilidad de la siembra del petróleo. El siglo petrolero venezolano ha sido un campo de disputa político-intelectual hegemonizado por el nacionalismo petrolero sin que hayan estado ausentes los actores que presionan hacia la privatización extranjerizante del.

\section{La política económica del petróleo y el auge del siglo petrolero venezolano}

\section{De Gumersindo Torres a Arturo Uslar Pietri}

El reconocimiento del derecho de los propietarios del suelo siquiera a la preferencia para obtener de la nación la facultad de explotar el subsuelo, mediante el pago de los impuestos legales, valorizaría enormemente la propiedad territorial, porque los agentes de las compañias tendrían que entenderse previamente con ellos para obtener esa facultad (...) La valorización de la propiedad territorial se traduciría naturalmente en la prosperidad general. (G. Torres citado en Baptista y Mommer, 1992, p. 11)

Con estas palabras en la Memoria del Ministerio de Fomento de 1920, en ese momento bajo su cargo, iniciaba Gumersindo Torres una larga tradición de pensamiento sobre política económica del petróleo en Venezuela. La política económica del país, basada en la Ley de minas de 1909, procuraba no poner ningún impedimento al capital interesado en la explotación minera, exonerándolo de cualquier presión impositiva especial (Egaña, 2010, p. 373; Mommer, 2010, pp. 73-76). El inicio, en 1914, de la disputa decisiva entre los Estados Unidos y Alemania por sustituir a Gran Bretaña como potencia hegemónica de la economía-mundo capitalista deparó un aumento de la demanda mundial de petróleo, por lo que para 1920 el petróleo se convirtió en el recurso natural más importante de Venezuela ${ }^{3}$.

En el medio de este proceso histórico-mundial, Gumersindo Torres (citado en Baptista y Mommer, 1992, p. 10) plantearía una distinción vital para el devenir de la estructura económica de Venezuela: "Son nociones distintas la de impuesto y la de percepción de una suma derivada de estipulación contractual

3 "Venezuela tuvo, desde el arribo de los primeros adelantos castellanos, una economía de plantación. El añil y el cacao con los cueros primero y después el café significaron la espina dorsal de esa economía (...) Cada región donde se han implantado esos cultivos, tiene más acceso a Europa y a Norteamérica que a comarcas del resto del país” (Rangel, 2004, pp. 46-47). 
por el goce de una propiedad nacional (...) En Venezuela hay impuestos, pero nada pagan las empresas por el derecho mismo de la explotación”. Torres le otorgaba al petróleo, en cuanto mercancía que se valoriza en el mercado mundial, un rol central para la prosperidad económica del país a partir de un "canon de arrendamiento" en terrenos privados y un "impuesto" en la propiedad del Estado (Mendoza Potellá, 2011, p. 39). El camino iniciado por Torres implicó la participación nacional en las "utilidades" del petróleo, estableciendo una identidad entre intereses nacionales, ingreso petrolero y prosperidad general.

Al mismo tiempo, el presidente del Banco de Venezuela, Vicente Lecuna, comparando la situación petrolera del país con los Estados Unidos (EUA), argumentaba que la especificidad venezolana era que la producción petrolera iba directo a la exportación (Mommer, 2010, p. 82). La consecuencia directa para la política petrolera del país de la propuesta de Lecuna es la apropiación por parte del Estado del ingreso rentista, independientemente de la ubicación de los yacimientos. Tanto para Gumersindo Torres como para Vicente Lecuna el ingreso rentista era el único beneficio que podría obtener el país de la explotación petrolera, ya que se carecía de capital y tecnología proporcional a la dimensión de los yacimientos ${ }^{4}$. Sus diferencias radicaban en la posterior distribución del ingreso o cuarto eje de la razón rentista. Mientras que Torres buscaba favorecer a los terratenientes directamente, Lecuna buscaba favorecer a la burguesía indirectamente. Torres reivindicaba el derecho de los terratenientes a obtener concesiones y Lecuna argüía, valiéndose del decreto del Libertador Simón Bolívar del 24 de octubre de 1829, que era la república quien debía percibir el ingreso rentista. En ambas posturas ante la política económica del petróleo se sedimentaba la idea de una inserción dependiente y rentista de Venezuela en la división internacional del trabajo.

El 30 de junio de 1920 se aprobó la primera ley abocada exclusivamente a legislar al sector petrolero, la cual, en sintonía con el espíritu nacionalista de Torres, favoreció el ingreso nacional ejercido mediante las regalías, instaló el régimen concesionario y privilegió el derecho de los terratenientes (Lieuwen, 2016, p. 50). Sin embargo, por la creciente inconformidad de las empresas petroleras y la falta de inversiones dirigidas a la explotación petrolera, en 1922 se destituyó a Torres del ministerio de Fomento y se aprobó una segunda ley petrolera (Mendoza Potellá, 2011, p. 40). Al tiempo que Gómez y sus allegados acrecentaban su patrimonio de manera inimaginable mediante el tráfico de concesiones, el pozo Barroso II reveló el potencial petrolero: "Venezuela, productor insignificante en 1922, se convirtió en 1928 en el principal exportador y segundo productor mundial, después de los Estados Unidos" (Lieuwen, 2016, p. 70).

4 "Debido a la precariedad económica y técnica del país, el Estado venezolano no se hallaba en condiciones de actuar como empresario en el negocio petrolero. Por tal motivo (y como expresión de los intereses extranjeros y de la oligarquía terrateniente) el Estado abre las puertas al capital foráneo en condiciones sumamente favorables y entreguistas" (Rodríguez 2015, p. 134).
La razón rentista y sus teóricos: la economía política en el siglo petrolero venezolano

Malfred Gerig 
Dossier América Latina: Venezuela

A finales de la década de 1920 la dinámica económica de Venezuela ya se encontraba en plena conexión con la del mercado mundial ${ }^{5}$. El hundimiento especulativo de Wall Street a finales de 1928 produjo una ola mundial de protección de monedas que culminó con "la suspensión de la convertibilidad en oro de la libra británica en septiembre de 1931" (Arrighi, 1999, p. 329). El mismo camino siguió el dólar estadounidense en 1933. El tipo de cambio USD/Bs pasó de cotizarse de 5,2 en 1912 a 7,56 en 1932 para retornar a 3,06 en 1934 (Lieuwen, 2016, p. 123). En medio de esa encrucijada del sistema monetario internacional Vicente Lecuna tuvo un segundo rol decisivo en la historia de la política económica del petróleo en Venezuela cuando, en 1934, debatió con Alberto Adriani en torno a la devaluación del bolívar. Adriani advertía sobre las profundas consecuencias de esta revalorización del bolívar para la agricultura venezolana: "con el nuevo dólar desvalorizado, o lo que es lo mismo, con el bolívar caro, los precios de nuestros productos de exportación se han hecho irrisorios" (Adriani citado en Baptista y Mommer, 1992, p. 15). Para confrontar la situación Adriani propuso devaluar el bolívar hasta hacer competitivas las mercancías de exportación tradicionales, especialmente el café. Lecuna, por su parte, argumentó que la devaluación favorecía a las compañías petroleras, ya que implicaba pérdidas que no eran comparables con la reactivación de las exportaciones agrícolas (Baptista y Mommer, 1992, pp. 15-16). En el argumento de Lecuna pesaba el segundo eje de la razón rentista, esto es, la maximización de los ingresos petroleros, ya que predominaba "el criterio de que mientras más bajo fuese el tipo de cambio del dólar con respecto al bolívar, mayor sería el ingreso de divisas por este concepto" (Crazut, 2010, p. 148).

Por intermediación de Lecuna, en 1934, se signó el "Convenio Tinoco", régimen cambiario donde "las compañías petroleras (...) convinieron en vender dos terceras partes de sus dólares a los bancos venezolanos a 3,90 bolívares (los bancos los venderían a 3,93). Los restantes dólares (...) convinieron las compañías en venderlos al gobierno al precio de importación del oro (3,06 bolívares)" (Lieuwen, 2016, p. 124). El Convenio Tinoco privilegió el escenario nacional donde los gastos crecientes en bolívares de las empresas petroleras y la abundante oferta de divisas presionaban hacia la revaluación del tipo de cambio, por encima de un escenario internacional caracterizado por las devaluaciones competitivas, los controles de cambio y la ruptura del patrón oro centrado en la City de Londres. La súplica de Adriani no fue oída por la vía de la devaluación del bolívar, sino que en 1936 se instaló un sistema de subsidios a las exportaciones agrícolas que oscilaba por los 10.000.000 de Bs anuales (Lieuwen, 2016, p. 124; Crazut, 2010, p. 138).

Así, el Convenio Tinoco marcó los derroteros del siglo petrolero venezolano en cuanto que la sobrevaluación del bolívar, que dificulta las exportaciones

5 "Año a año los hidrocarburos fueros descontando ventajas a las exportaciones tradicionales, hasta llegar a 1926, cuando logran desplazarlas del primer plano del mercado externo" (Rodríguez, 2015, p. 101). 
no-petroleras y fomenta las importaciones baratas, se convirtió en el eje de la política cambiaria. Al reclamo de la soberanía del Estado sobre el petróleo logrado mediante el sistema de concesiones de 1920, le sucedía la necesidad de maximizar el ingreso petrolero que subyacía al Convenio Tinoco.

Tras la muerte de Gómez, en la voz de Arturo Uslar Pietri surgía una conciencia crítica sobre las consecuencias que el petróleo acarreaba para la economía del país. Recogiendo motivos planteados por Adriani y partiendo de una concepción del petróleo como agente momentáneo y destructivo, Uslar Pietri (2006, p. 22) planteará la primera versión acabada de la "razón rentista" bajo la metáfora "sembrar el petróleo": "Urge aprovechar la riqueza transitoria de la actual economía destructiva (...) Es menester sacar la mayor renta de las minas para invertirla totalmente en ayudas, facilidades y estímulos a la agricultura, la cría y las industrias nacionales". Para Uslar la política económica del petróleo cobra un imperativo moral, esto es, convertir una riqueza efímera y destructiva en los cimientos para una reproducción productiva de la sociedad, empezando por el desarrollo de la agricultura. Allí hace aparición el tercer eje de la razón rentista: la posibilidad de invertir los ingresos rentistas en un proyecto nacional de desarrollo.

La llegada al poder de Eleazar López Contreras, ministro de guerra de Gómez, significó el rejuvenecimiento del debate sobre la renta petrolera. Al gobierno se le presentó una encrucijada, según la cual o la renta "podía convertirse directamente en bienes de consumo" o "podía transformarse en capital productivo" (Baptista, 2008, p. 284). Uslar Pietri, quien, junto a M.R. Egaña y Adriani, representaba las posiciones más acabadas sobre el asunto, fomentó el desarrollo entendido como inversión reproductiva de la efímera riqueza petrolera, teniendo siempre como argumento las consecuencias destructivas que el gasto en consumo puede acarrear. Sin embargo, es con Uslar que nace una nebulosa metafísica que borra las condiciones objetivas de la inserción de Venezuela en la división internacional del trabajo a favor de argumentos estrictamente morales, los cuales desplazan el debate de las características de la economía venezolana hacia un asunto de voluntad sin tener en cuenta los determinantes "externos", es decir, la estructura polarizada de la economía-mundo capitalista.

\section{Juan Pablo Pérez Alfonzo y la imposible siembra del petróleo}

La dificultad consistía en reconocer que ese tipo de riqueza no ganada, porque no se generaba por actividades propiamente dichas, en su mayor parte consistía en la liquidación de un activo preexistente, tenía que inyectarse a la economía como peligroso medicamento. (Alfonzo, 2011, p. 40)

Esto argumentaba Pérez Alfonzo, refiriéndose a los debates sobre la política económica del petróleo que se generaron en 1936 tras la muerte de Juan Vicente Gómez, cuyo legado económico fue la implantación del predominio absoluto del petróleo en la inserción del país en el mercado mundial y la di-
La razón rentista y sus teóricos: la economía política en el siglo petrolero venezolano Malfred Gerig 
Dossier América Latina: Venezuela visión internacional del trabajo ${ }^{6}$. Con el Programa de Febrero presentado en 1936 por el presidente Eleazar López Contreras, forjado en gran parte por Manuel R. Egaña, se vuelve "sentido común" una voluntad desarrollista que, obviando las efectuaciones de la inserción del ingreso petrolero en la estructura económica del país dadas las características de la mercancía petróleo en su origen, se preocupa en maximizar la renta petrolera como palanca del desarrollo (Baptista, 2008, pp. 282-283).

La “inyección”, como metáfora utilizada por Pérez Alfonzo, era una crítica al desarrollismo que escindía el origen de la mercancía petróleo con el destino de la renta que se captaba del mercado mundial, es decir, la economía política del petróleo escindida de la política económica petrolera. Según los argumentos desarrollistas "se considera al capital extranjero como un elemento positivo y favorable para el país...", mientras que de acuerdo con las explicaciones esgrimidas por Pérez Alfonzo (1971, p. 90) "la mecánica del capitalismo tiende a que las inversiones extranjeras expolien a los países atrasados que la reciben". Con la tesis de la dependencia se produce un quiebre de profundas consecuencias en la tradición de la política económica del petróleo en Venezuela hasta el punto de partir en dos al nacionalismo petrolero.

Durante el periodo 1936-1945 se ocasiona una galvanización de procesos económicos y sociales a escala sistémica y nacional donde el conflicto social transitó los tres momentos de las correlaciones de fuerza señalados por Gramsci (2004, pp. 414-416), teniendo sus puntos máximos de ebullición en la reforma petrolera de 1943 y el golpe de Estado del 18 de octubre de 1945. En el plano sistémico se desarrolla la disputa de los años treinta entre EUA y Alemania por la sustitución de Gran Bretaña en el puesto de mando del sistemamundo moderno que tendrá su fase culminante de 1939 a 1945 (Gerig, 2019, p. 119). La exportación de petróleo de EUA a Europa marcaba la diferencia a favor de los aliados en una gran guerra de coalición nunca antes vista. La expansión del conflicto causada por el ataque japonés a Pearl Harbor y la ofensiva de los submarinos alemanes para interrumpir el transporte y la cadena de suministros petroleros por el océano Atlántico (Yergin, 1992, p. 492) ocasionó una minicrisis petrolera en Venezuela, caracterizada por el sobre inventario del almacenamiento, la disminución de la producción y la paralización de las nuevas inversiones. Para el fisco venezolano el resultado fue la reducción de

6 "Cuando muere Gómez, a quien sostiene el petróleo en los últimos quince años de su gobierno, el país ya produce cerca de trescientos mil barriles diarios. En el escalón de los productores de la codiciada sustancia nos corresponde el segundo lugar. Venezuela ha desplazado a Rusia y México de esa alta categoría. Somos el primer exportador mundial, con fuertes colocaciones en Europa que es nuestro principal cliente de entonces, y en los Estados Unidos. Las inversiones petroleras llegan ya a cerca de cuatrocientos millones de dólares. Detrás de Cuba y Chile es la Venezuela de esa época el primer campo de colocación de capitales para Estados Unidos. Absorbemos más importaciones por habitante que cualquier otro país de América Latina. Y tenemos el presupuesto más alto del continente en función del número de habitantes del país" (Rangel, 2006, pp. 210-211). 
los impuestos captados de las compañías "a 67.000 .000 de bolívares en el año fiscal terminado el 30 de junio de 1943, una tercera parte menos que el año anterior, y los ingresos aduaneros, que dependían considerablemente de la actividad petrolera, se redujeron a 88.000.000, $44 \%$ menor que en la preguerra" (Lieuwen, 2016, p. 162). Las correlaciones de fuerzas sociales estaban dadas para que en el plano de las correlaciones de fuerzas políticas se instalara un clima de reforma.

La irrupción de la Generación del 28 puso en evidencia fisuras en la organización política del país surgida en 1902-1903. Sin embargo, los espontáneos nuevos grupos aspirantes a clase política eran aún incapaces de plantear el conflicto en el plano universal/estatal, esto es, instalar una polémica histórica capaz de llevar las demandas del plano corporativo a la sociedad civil. Es la muerte de Juan Vicente Gómez (1935) el acontecimiento que abrió la posibilidad para la competencia política en el plano universal/estatal. La revuelta popular de 1936 reveló en el orden gomecista contradicciones insanables, abriendo un periodo de equilibrio inestable que se prolongó hasta $1958^{7}$. La reacción del pos-gomecismo encarnado en López Contreras ante la revuelta se da mediante la asunción de un programa de reformas que polarizó el tablero político entre reforma y revolución, entre la Revolución de Octubre y el New Deal (Rangel, 2004, p. 101). No obstante, es con la ley petrolera de 1943 que la abstracta "tergiversación de posiciones" reformistas da lugar al concreto conflicto entre dos nacionalismos petroleros, entre un programa desarrollista y la crítica a la dependencia. El 18 de octubre el conflicto escala al decisivo plano militar.

Por un lado, se encontraba la aceptación -surgida a partir del debate Gumersindo Torres-Vicente Lecuna-, según la cual el país no podía integrarse en la economía mundial como productor petrolero, correspondiéndole la justa reivindicación por la propiedad del recurso. El "nacionalismo petrolero primero" se centraba en legitimar la reivindicación rentística nacional como paso previo a una maximización del ingreso rentista que conduciría al desarrollo ${ }^{8}$.

7 "El ascenso de masas de 1936, fue quizá uno de los más completos que haya registrado Venezuela. No sólo se extendió sobre toda la geografía nacional (...) sino que ganó a todas las capas de la población. Desde los artesanos de informe conciencia hasta la pequeña burguesía local (...) cada colectividad vibró íntegramente en una especia de despertar político" (Rangel, 2006, pp. 290-291).

8 La ley "unificó la legislación petrolera. Antes de que la ley entrara en vigor, las compañías tenían que adaptarse a ella, y cumpliendo sus promesas lo hicieron inmediatamente. La nueva ley canceló todas las viejas deudas, reclamaciones y disputas sobre las antiguas concesiones, ya que sustituía a toda la legislación anterior. Los derechos y deberes de todos los concesionarios eran ahora los mismos. Lo más importante para el Estado, naturalmente, fue el gran aumento de su participación en los beneficios de la industria (...) Se esperaba que los nuevos impuestos incrementasen los ingresos nacionales procedentes del petróleo, aproximadamente, en $80 \%$, y se previó que los ingresos del tesoro por este concepto igualarían ahora los beneficios de la industria. También se fomentó el refinado en el país y se autorizó al presidente para ofrecer concesiones de cincuenta años, exenciones de derechos aduaneros y reducciones de impuestos a los 
Dossier América Latina: Venezuela

Para Mommer y Baptista (1992, p. 28) "ello llevó al enmascaramiento de esta reivindicación como una transacción entre iguales, a saber, entre capitales que sólo diferían en su nacionalidad. A tal fin se imponía la definición del recurso natural en cuestión como capital". Por otro lado, el "segundo nacionalismo petrolero" no dudaba que Venezuela pudiese convertirse en un país productor de petróleo. En este caso, la legitimación del ingreso rentista se sostenía en la denuncia a la expoliación por parte del capital internacional. El drenaje de recursos se vería contrarrestado en sus intereses con una política de maximización del ingreso petrolero captado por el país para luego ser distribuido directamente en la población?.

A diferencia de la vertiente desarrollista que definía al recurso como un capital, la crítica dependentista que se desprende de Betancourt y Pérez Alfonzo caracterizó al petróleo como un recurso natural no renovable por lo que "los yacimientos petrolíferos no eran conmensurables ni con el capital ni con el trabajo" (Baptista y Mommer, 1992, p. 37). El objetivo de caracterizar así a la naturaleza económica del petróleo no estaba motivado por dilucidar rigurosamente la diferencia especifica de esta mercancía dentro de la economía-mundo capitalista para con ello desentrañar las consecuencias que cierta política petrolera podía tener sobre la estructura económica de Venezuela. Al contrario, en sintonía con la razón rentista, su intención última era la captación de cada vez más plusvalor del mercado mundial por el concepto de renta internacional del petróleo.

La Ley de Hidrocarburos de 1943 le dio una segunda vida al régimen de concesiones, extendiéndolo por 32 años (con reformas muy parciales) hasta la nacionalización de la industria petrolera en 1976. Una vez que Acción Democrática (AD) arribó al poder tras el golpe de Estado del 18 de octubre de 1945, Pérez Alfonzo en su condición de Ministro de Fomento atemperó la reivindicación nacionalista, decidiendo mantener la ley de 1943 y enfocar la política petrolera hacia la formula fifty-fifty y las relaciones entre el capital y el trabajo en la industria (Lieuwen, 2016, p. 183). La política petrolera de Pérez Alfonzo no se daba en el escenario de 1940, donde el 85\% del petróleo que consumía Europa llegaba de EUA, sino en un escenario donde Oriente Medio proveía el 85\% del petróleo consumido en el Viejo Continente (Panitch y Gindin, 2015, p. 161). Además, la expansión económica de la posguerra intensificó la pauta

que refinaran en el país" (Lieuwen, 2016, p. 170).

9 El conocido "Voto Salvado" de Pérez Alfonzo ante la ley de 1943 sostenía: "En una síntesis general del proyecto podrían señalarse dos grandes grupos de ventajas (...) La unificación de las relaciones de los concesionarios con el Estado; y la definida y clara intervención que en el funcionamiento de la industria se reconoce al mismo Estado. Entre las de orden económico, parecen indiscutible el aumento apreciable de los impuestos, tomados en conjunto, y no obstante la desaparición de impuestos que antes pagaban los concesionarios (...) Se pretende que las ventajas señaladas signifiquen la cancelación del pasado (...) Daños cuya reparación no puede ser borrada por la simple disposición de una ley" (J. P. Pérez Alfonzo citado en Mendoza Potellá, 2011, pp. 49-50). 
intensiva en energía y medios de producción de la senda de desarrollo occidental $^{10}$. En los países petroleros la "poderosa correa de transmisión de la revolución social, que durante y después de la guerra se extendió a todo el mundo no-occidental bajo la forma de movimientos de liberación nacional" (Arrighi, 1992, pp. 84-85) tomó concreción con la lucha por la maximización del ingreso rentista a través del fifty-fifty.

El aumento del ingreso rentista fue conducido durante el trienio 19451958 a la inserción agotadora, inaugurando el modelo de desarrollo en el cual la inserción del ingreso rentista vía empleo público fomenta la demanda agregada, y esta, a su vez, presiona a la expansión del mercado de trabajo y el mercado interno (Baptista, 2010, p. 210). El Trienio Adeco puso en escena el cuarto eje de la razón rentista: el conflicto político por la distribución de la renta internacional del petróleo.

Si el golpe de Estado del 18 de octubre de 1945 llevó el conflicto al plano militar, el golpe de Estado del 24 de noviembre de 1948 demostró que aún no se había agotado históricamente el viejo orden político nacido en 1902-1903. La junta militar y la posterior dictadura de Marcos Pérez Jiménez, que gobernó Venezuela de 1948 a 1958, se asentó en el statu quo sin modificar en demasía la política de inserción de la renta petrolera, sin embargo, sí atentaron contra las conquistas obreras del Trienio Adeco y quebraron la política de no más concesiones (Lieuwen, 2016, p. 193). El 23 de enero de 1958 se cierra la etapa de las correlaciones de fuerza militares con el nacimiento de la hegemonía de AD. Quedó configurada la escena política que regirá la segunda mitad del siglo XX. Por un extremo, los representantes de la vieja sociedad desaparecen paulatinamente. En el centro, una vital Acción Democrática nuclea a la sociedad política, siendo también el partido del extranjero. A su derecha se encuentra COPEI como avatar del bipartidismo excluyente. Por el otro extremo está la novísima izquierda, intentando convertirse en la principal fuerza social contrahegemónica.

Una vez que Pérez Alfonzo se convierte en ministro de Minas e Hidrocarburos, pone en marcha el programa de política petrolera más ambicioso y coherente de la historia del país. Con el pentágono petrolero la razón rentista se convirtió en programa de gobierno. El reclamo de la soberanía sobre el recurso ya no se planteó en términos de un reconocimiento del Estado como propietario, sino como la necesidad de que este se convierta en productor. Para ello, el principio de no más concesiones y la creación de la Corporación Venezolana de Petróleo (CVP). La maximización del ingreso rentista era el centro de la política petrolera. Para ello se implementó el principio de no más concesiones y se creó la Comisión Coordinadora de la Conservación y el Comercio de los Hidrocarburos y la Organización de Países Exportadores de Petróleo (OPEP).

10 "Nadie dentro del sector petrolero estaba preparado para tamaña explosión de la demanda de todos los productos petrolíferos. Las ventas de gasolina en Estados Unidos eran un cuarenta y dos por ciento mayores en 1950 que lo que habían sido en 1945" (Yergin, 1992, p. 540). 
Dossier América Latina: Venezuela
La "política pasiva -limitada a buscar como recaudar las superganancias existentes- no representaba sino una primera etapa en la historia del petróleo en Venezuela" van a señalar Baptista y Mommer. "La segunda etapa, en cambio, habría de caracterizarse por una política petrolera activa, con la cual el Estado propietario arbitraría los medios y las vías para influir sobre mecanismos del mercado, aumentar esas superganancias y apropiárselas de seguida" (Baptista y Mommer, 1992, p. 44). El segundo nacionalismo petrolero llevó la razón rentista a su máxima expresión hasta que consuma su programa histórico con la nacionalización de la industria.

Sin embargo, la nacionalización coincide con el agotamiento de la fase de expansión material del siglo petrolero venezolano y su "crisis señal". Pérez Alfonzo será uno de los primeros en denotar que el país ingresó en un periodo de incapacidad para la absorción "productiva" de la enorme masa de capital en la forma de dinero que capta del mercado mundial:

Quizás entre los hechos ocurridos de mayor significación para la política petrolera está la experiencia vivida por el país que muestra las limitaciones del crecimiento económico en Venezuela. Quedó demostrado el principio, por lo demás evidente, de que existe una capacidad relativamente fija para emplear útilmente nuevos capitales. En cuanto se extralimita esa capacidad de absorción de capital, el crecimiento económico deja de corresponderse a las mayores cantidades empleadas más allá de aquellos límites. Entonces, dicen algunos, se trata de la inundación de capital, mientras otros los efectos de la indigestión económica. (Pérez Alfonso, 1971, p.18)

Pérez Alfonzo abordó la cuestión de la dependencia en un sentido original, ya que no se refiere a la dependencia de Venezuela como país periférico en la división internacional del trabajo. Para él, la "dependencia” es de la economía y la sociedad venezolana con respecto al petróleo a la hora de reproducir la riqueza y el crecimiento económico. De ahí que a medida que incrementa el ingreso que circula hacia Venezuela proveniente del sistema capitalista la estructura económica interna, de ninguna forma autárquica sino inserta en un sistema global de acumulación de capital, tiende a ser incapaz de absorber productivamente dichos recursos por lo que busca constantemente fugarlos hacia el mercado mundial.

Utilizando una retórica apesadumbrada y con algunas rémoras moralistas que ofuscan en menor grado lo que sus palabras querían sostener, Pérez Alfonzo (2011, p. 179) señaló la pauta que la economía política del petróleo habría de tener presente en la fase de declinación del siglo petrolero venezolano al indicar como invertir productivamente el petróleo era un trabajo de Sísifo: "El dinero ofusca al extremo de hacernos impermeables a toda la dolorosa y larga experiencia en afanoso pero inútil empeño en una supuesta pero imposible siembra del petróleo". 


\section{La economía política del petróleo y la caída del siglo petrolero venezolano}

\author{
Arturo Uslar Pietri y el capital depositado por la naturaleza \\ en el subsuelo
}

La razón rentista y sus teóricos: la economía política en el siglo petrolero venezolano

Malfred Gerig

El petróleo no es ni una cosecha ni una renta, sino el consumo continuo de un capital depositado por la naturaleza en el subsuelo. Todo capital que se consume tiene un término. Ese término de la riqueza petrolera, de la que estamos viviendo, es la más trágica interrogante que surge en el panorama de nuestro futuro económico y social. (Uslar Pietri, 2006, p. 32)

La correcta elucidación de las determinantes de un fenómeno económicosocial no se constriñe a un problema hagiógrafo en el que compiten distintas ideas, todas ellas escindidas de alguna implicación práctica en el mundo en que se producen. De ahí que, lo que Baptista denominó la "recta caracterización” del petróleo como mercancía que se intercambia en el mercado mundial es un problema conceptual con profundas implicaciones prácticas ${ }^{11}$ : la economía política del petróleo fundamenta a la política petrolera. Capital, suelo o trabajo generan un tipo de rédito ${ }^{12}$. Arturo Uslar Pietri fue un pensador que de la mano de la política petrolera cruzó de manera precursora al campo de la economía política del petróleo.

Al momento de caracterizar económicamente al petróleo, Uslar acogió la vía de la negación: "no es ni una cosecha, ni una renta". Para Uslar la riqueza que emana del petróleo no pertenece a aquellos ingresos cotidianos que forman la riqueza de las naciones: el trabajo (Baptista, 2008, p. 264). "Es menester -sostendrá en su clásico Sembrar el petróleo- sacar la mayor renta de las minas para invertirla..." (Uslar Pietri, 2006, p. 22), claramente llamando "renta”, según el uso anglosajón, a los ingresos petroleros en la forma de dinero en lugar del tipo de rédito que una renta internacional del petróleo producía. $\mathrm{Al}$ acometer la definición de la mercancía petróleo desde el plano positivo arguye "...el consumo continuo de un capital depositado por la naturaleza en el subsuelo" (Uslar Pietri, 2006, p. 32). El problema de investigación de Uslar no se encontraba en la definición del petróleo, sino en la forma correcta de invertirlo, por lo que -en búsqueda de una postura en política económica del

11 “Todo el conocimiento posible acerca del petróleo depende de su recta caracterización como mercancía que se intercambia en el mercado mundial. Pero también depende, de esta recta caracterización, toda práctica y toda política económica que se intenta llevar adelante" (Baptista, 2008, p. 262).

12 "En capital-ganancia o, mejor aún, capital-interés, suelo-renta de la tierra, trabajo-salario, en esta trinidad económica como conexión de los componentes del valor y de la riqueza en general con sus fuentes, está consumada la mistificación del modo capitalista de producción, la cosificación de las relaciones sociales, la amalgama directa de las relaciones materiales de producción con su determinación histórico-social” (Marx, 1985, p. 1056). 
Dossier
América Latina:

Venezuela

petróleo- minó el rigor con el que se debía atender la cuestión de la economía política, de la recta caracterización. Acudió al concepto "capital", ya que este le otorga la posibilidad de introducir la cuestión de la depreciación y la inversión desde un plano estrictamente moral.

Situado desde un tipo ideal de inversión del ingreso que percibe el país al intercambiar petróleo en el mercado mundial, Uslar proyecta la razón rentista sobre su definición del petróleo como mercancía. La categoría "capital depositado por la naturaleza en la tierra" conduce a equívocos analíticos visibles en aspectos claves como la relación entre petróleo y valor o el entendimiento del capital como una relación de producción y no como una cosa. No obstante, esto le sirve para posicionar una boya de flotación constante a lo interno del pensamiento sobre el petróleo en Venezuela: la necesidad de invertir certeramente el ingreso petrolero, sembrar el petróleo. En resumen, Uslar plantea una agonística de la inversión petrolera en detrimento de una correcta economía política del petróleo.

\section{Bernard Mommer y la teoría de la renta internacional del suelo}

Podemos decir que encontramos una renta internacional del suelo de manera palpable en los países petroleros. Pero lo que debe entenderse por este concepto no ha sido tratado nunca por la literatura teórica conocida (...) La teoría de la renta de Marx debe ser reexaminada en este contexto. (Mommer, 2010, p. 24)

Partiendo de la estela dejada por Marx en el libro tercero de El capital donde se ocupó de la cuestión de los réditos y sus fuentes (Marx, 1985, p. 1037), Bernard Mommer recupera para el análisis del tipo de ganancia que origina la explotación petrolera a la categoría "renta del suelo". El autor sostendrá que la categorización equívoca del petróleo como "capital natural" tiene su origen en David Ricardo y Alfred Marshall. Según la consideración teórica de Ricardo, la renta del suelo refiere a un valor intrínseco del producto de la tierra. Ricardo llegó a esta conclusión al afrontar el problema de la relación entre valor y precios, ya no de la agricultura, sino de la industria extractiva. La teoría del valor-trabajo ricardiana al realizar una identidad entre valores y precios se muestra incapaz de explicar un sobreprecio originado en la propiedad del recurso. Por lo que la categoría "valor intrínseco" se convierte en un subterfugio teórico en contradicción directa con la teoría del valor-trabajo que el mismo Ricardo preconizara.

En contradicción con el esbozo de Ricardo, Mommer se adentra en la problemática a través de las categorías marxianas de "renta absoluta" y "renta diferencial". Sucintamente, la renta absoluta se refiere a la ganancia extraordinaria que la propiedad territorial le carga en la forma de "renta" a las tierras de menor productividad y que, al expresarse en los precios, "recae sobre toda la clase capitalista" (Mommer, 2010, p. 16) directamente y, en la clase trabajadora, indirec- 
tamente. Por su parte, la renta diferencial describe las ganancias extraordinarias de las que se apropia un terrateniente cuya propiedad goza de mayor productividad con respecto a otra (Mommer, 2010, p. 24). Así pues, la cuestión de la propiedad pasa a jugar un papel central en la determinación de la renta del suelo en sus dos formas: industria extractiva o agricultura. De ahí que:

En lo que a petróleo se refiere, observamos que dentro del mundo capitalista se encuentra tanto la propiedad privada como la propiedad estatal de los yacimientos (...) Empero, la definición que hemos dado hasta ahora de la propiedad estatal es insuficiente en el contexto internacional (...) Si los yacimientos nacionales estatales son objeto de una explotación internacional, puede darse la tendencia en la clase dominante nacional, como propietaria común y ocupante del territorio nacional, a considerar estos yacimientos como propiedad nacional, con lo cual el Estado puede aspirar a cobrar una renta lo más alta posible sobre la producción a exportar. (Mommer, 2010, p. 20)

Mommer introduce un desplazamiento en la "unidad de análisis" de la cuestión petrolera al sostener que la categoría renta del suelo no puede explicarse en un contexto nacional. Un paso -quizá insuficiente, pero relevantecontra el nacionalismo metodológico. La extracción de minerales y petróleo es siempre una industria, ya que presupone cadenas de valor globales en la división internacional del trabajo. La renta se sostiene en una de las condiciones fácticas del capitalismo histórico: la división internacional del trabajo. La acumulación de capital divide la territorialidad del trabajo, creando cadenas de valor globales regidas por la centralización del plusvalor entre clases, regiones y Estados (Wallerstein, 2004).

Pese a que en su exposición Mommer todavía carece del aparataje teórico para explicitar la dinámica del plusvalor a nivel global, sí hace significativos avances a la hora de esclarecer que la propiedad nacional-estatal puede gozar, mediante la renta internacional del suelo, de una porción del plusvalor que se crea en la economía-mundo capitalista.

En lo que respecta a la propiedad nacional-estatal sobre el petróleo, Venezuela es un ejemplo de primer orden: "sólo en el caso de Venezuela encontramos la transformación de la propiedad estatal, desde la libre propiedad a la propiedad estatal nacional completamente desarrollada en todas sus etapas" (Mommer, 2010, p. 22). Lo que permitió la nacionalización del capital arrendatario a través de la maximización del ingreso rentista. La caracterización del tipo de ingreso que origina la mercancía petróleo que la teoría de la renta internacional del suelo plateada por Mommer da significativos pasos en pro de la clarificación analítica del problema. Con Mommer la economía política del petróleo recobra terreno, por muy pesimista que dicha caracterización pueda ser en materia de política económica del petróleo. A partir de la década de 1989 el piso sólido donde se movió la razón rentista durante más de cincuenta años empieza a resquebrajarse en la práctica y también en la teoría.
La razón rentista y sus teóricos: la economía política en el siglo petrolero venezolano

Malfred Gerig 
Dossier
América Latina:

Venezuela

El autor les vaticinaba "la decadencia a largo plazo de sus rentas petroleras" (Mommer, 2010, p. 310) a los países que habían gozado de un intercambio desigual a favor, a través de la renta, para apalancar su acumulación nacional de capital. De igual forma advertía sobre las profundas transformaciones socioeconómicas que este ocaso traería. Tras la belle époque del siglo petrolero venezolano (2004-2012) estas palabras han dejado de ser una lejana posibilidad para que esas transformaciones sacudan sus consecuencias sobre la sociedad venezolana. Venezuela ha llegado al final de su ciclo petrolero, por lo que no existe ninguna posibilidad de conseguir equilibrios estáticos dentro de la matriz de la razón rentista. La economía venezolana se enfrenta a una encrucijada de destrucción creativa (Schumpeter, 1969, p. 118) donde o modifica cualitativamente su inserción en la división internacional del trabajo o transita rápidamente de la crisis al colapso y del colapso a la extinción.

\section{Asdrúbal Baptista y el capitalismo rentístico}

¿Qué forma característica adquiere en su caso la generación del excedente que en importante medida lo mueve y dinamiza? Se ha visto ya cómo su realidad descansa sobre un hecho básico, a saber, la captación por el Estado, que es propietario de un medio de producción no producido, de un ingreso o provento que le paga el mercado mundial con cargo a su propiedad. (Baptista, 2010, XXXI)

Con los trabajos de Asdrúbal Baptista el pensamiento abocado a la economía política del petróleo realiza un salto superlativo. Su teoría del "capitalismo rentístico" se enfoca en resolver de manera holística la cuestión de la renta petrolera tanto en el origen como en el destino. Con su proceder teórico se abre un juego de categorías analíticas que hacen inteligible la caracterización del petróleo. Procedamos, siguiendo el dictum de Hegel según lo cual "lo verdadero es el todo", a adentrarnos en la teoría de Baptista.

Para Baptista el marco en el que la mercancía petróleo cobra sentido práctico y analítico es el mercado mundial. De ahí que no podamos encontrar respuestas a la cuestión petrolera si no ampliamos territorialmente nuestro marco categorial. La pregunta por la economía política del petróleo es tan sólo un eslabón de la pregunta sobre la naturaleza y causa de las riquezas de las naciones. "La especificidad del capitalismo rentístico -argumentaradica peculiarmente en el hecho de que su estructura abarca desde el inicio un doble ámbito espacial (...) y sólo desde esa dualidad es de donde se hace posible comprender históricamente su dinámica" (Baptista, 2010, p. XXXI). Encontramos así una postura que intenta "superar" el nacionalismo metodológico. La dialéctica entre Estado-nación y mercado mundial configura una doble espacialidad en la que se da un intercambio que otorga como 
resultado un ingreso para el propietario de la mercancía petróleo. La cuestión central de la economía política del petróleo es dilucidar la naturaleza de dicho ingreso.

Con esta finalidad, Baptista desempolvará una categoría olvidada en la economía política contemporánea: los medios de producción no producidos. En su consideración, a la propiedad le corresponde una remuneración cuando "concurre a la producción", sin embargo, en términos económicos, "hay dos clases de cosas que son objeto de posesión y propiedad” (Baptista, 2010, p. 13). En primer lugar, aquellos objetos que sirven y acuden a la producción, siendo originados en un proceso de producción pretérito: los medios de producción producidos. En segundo lugar, los objetos que participan en la producción sin ser resultado de una actividad productiva previa: los medios de producción no producidos. Categorizado como medio de producción no producido, el petróleo pasa a ser una mercancía antediluviana previa a toda historia económica. No obstante, Baptista (2010, p. XXXI) sacará consecuencias del procedimiento conceptual que lleva a cabo: "el carácter de excedente absoluto o incondicionado de ese provento, puesto que su contrapartida es un medio de producción no producido".

Con un exquisito eufemismo, la voz "excedente" viene a sustituir al termino plusganancia. El carácter de plusganancia lo otorgará, de acuerdo con el autor, la inexistencia de costos de producción para una mercancía que, como el petróleo, es un medio de producción no producido. En este momento del proceder analítico se diluye el llamado a expandir el ámbito territorial de análisis, así como la dialéctica producción-circulación en el mercado mundial.

En adelante, la explicación que se le consigue al excedente que se apropia el dueño de los medios de producción no producidos es el "simple ejercicio de un derecho de propiedad terrateniente" (Baptista, 2010, p. XXXI). Planteadas así las cosas, pareciese que la economía-mundo capitalista encontró en su desarrollo y expansión una mercancía central para la reproducción ampliada del capital que no estaba aún en posesión de capitalistas. Por lo que, dada su importancia, aceptó pagar una plusganancia a sus poseedores rentistas. El desarrollo histórico de la producción, el papel de la energía en la valorización del valor y el intento del capital por valorizarse con menor costo se difuminan. La propiedad se convierte en factor de mayor peso que la lógica de acumulación incesante de plusvalor para determinar la esencia del capitalismo histórico. Surge la pregunta: ¿Es el capitalismo un régimen de propiedad o un modo de acumulación?

La crítica es incapaz de difuminar el progreso que la recta caracterización del petróleo consigue con Baptista. Con su pensamiento se impone un fundamento insoslayable: en cuanto al ingreso que rige a la economía venezolana se trata de una "renta internacional del petróleo". Su definición: "la participación del propietario del recurso en unos ingresos excedentarios generados en el mercado de trabajo mundial (...) ese provento del propietario terrateniente no proviene del mercado de trabajo nacional" (Baptista, 2010, p. XXXI). La distinción entre
La razón rentista y sus teóricos: la economía política en el siglo petrolero venezolano

Malfred Gerig 
Dossier
América Latina:

Venezuela

mercado de trabajo "nacional" y "mundial" es un paso nunca dado con el rigor necesario por la tradición del pensamiento petrolero en Venezuela. En la tradición, el afuera ha sido siempre una instancia independiente que se relaciona con la economía nacional en la forma de dos totalidades autónomas. En el marco del doble ámbito espacial comentado, un excedente producido por el trabajo realizado en el mercado mundial ingresa en la forma de renta internacional a ser administrado por el Estado-nación en cuanto propietario. Las consecuencias y desbalances que de este hecho se desprenden marcarán la vida económica y social de Venezuela durante un siglo. El Estado rentista no debe regirse por la insoslayable relación entre productividad y salario real, sino que puede aumentar ficticiamente los niveles de consumo a través de importaciones que se soportan en un bolívar sobrevalorado. Con la voz "capitalismo rentístico" Asdrúbal Baptista acuña la especificidad de la vida económica venezolana:

Es capitalista, en cuanto una porción de su ingreso disponible total, de cuantía importante, se crea en la relación del capital a escala universal y allí se lo capta (...) Es rentística, puesto que la relación de origen de ese excedente se funda en una propiedad nacional ejercida por el Estado sobre un recurso no producido, al que demanda y requiere el mercado mundial. (Baptista, 2010, pp. XXXI-XXXII)

Que Baptista caracterice al petróleo como medio de producción no producido, incurriendo en una mistificación, no obsta para olvidar que ha sido él quien ha puesto en orden una serie de fundamentos que rigen el movimiento económico de la sociedad venezolana como parte del sistema histórico capitalista. No son óbice ciertos juicios mistificados para dejar de seguir lo que en su proceder teórico hay de revolucionario y clarificador. Con su proceder teórico la economía política del petróleo otorga unos cimientos capaces de hacer frente al ocaso del siglo petrolero venezolano y su correlato, la razón rentista.

\section{Conclusiones}

En un perspicaz estudio sobre Venezuela, Celso Furtado (1993, p. 259) sostenía en 1974 que "la acción del Estado venezolano está condicionada por las exigencias de una estructura económica que en gran medida refleja la forma como fue utilizado el excedente petrolero en el último cuarto de siglo". De 1973 a 1974 el precio del barril de petróleo aumentó de 3,29 a 11,58 dólares americanos. En ese escenario, el país ingresó en el gran intento histórico por sembrar el petróleo: la Gran Venezuela. Sin embargo, la libertad que otorgó al accionar económico del Estado el aumento del petróleo, por un lado, estaba estrictamente limitada por el metabolismo económico que había creado la inserción desde 1917 de una renta internacional del petróleo en las relaciones entre el sector público y el privado, el Estado y la sociedad 
civil, la economía venezolana y el mercado mundial. Para Furtado "se ha creado un sistema económico que produce poco excedente en la forma de ahorro e impuestos (...) y que obtiene poco rendimiento de las inversiones que el excedente petrolero permite realizar. Se trata de un sistema económico orientado fundamentalmente hacia el consumo y el desperdicio" (Furtado, 1993, p. 260).

En 1972, las importaciones de bienes y servicios como porcentaje del PIB se ubicaban en 19,9\%. En 1978, ascendieron hasta el 37,2 \%, para descender al $13 \%$ en 1983. Por su parte, el consumo final de los hogares como porcentaje del PIB comenzó un rápido ascenso desde el 45,5 \% en 1974 hasta el 78,65 \% en 1983 (BM, 2020). El aumento de los precios del petróleo desnudó la gran dificultad para absorber la renta internacional por parte de la economía nacional. La "lluvia de oro" de 1974 a 1978, para utilizar la frase de Furtado, sirvió para terminar de agotar la fase de ascenso material del siglo petrolero venezolano e instalar la fase de declinación, la cual se extendió desde 1983 hasta 2004. Luego de ascender al 51,8 \% en 1978, la formación bruta de capital no ha sobrepasado el $30 \%$ del PIB, teniendo un promedio de 21,06 \% desde 1983 hasta el 2004. Por su parte, el promedio del consumo final de los hogares (porcentaje del PIB) fue de $64 \%$ en el mismo periodo ${ }^{13}$.Venezuela transcurría lenta pero inexorablemente a través de la disminución de la composición organiza de capital de la base industrial nacional a un aumento del coeficiente de importaciones para subsanar la escisión entre producción y consumo. Inestabilidad del tipo de cambio, endeudamiento externo, fuga de capitales y crisis fiscal constituyeron el día a día de ese imperceptible proceso de disminución de la producción y la productividad.

Presagiando la dificultad para seguir engañando al futuro, Domingo Alberto Rangel sostuvo:

El siglo XXI plantea un dilema para nosotros que no podríamos evadir ni podríamos aplazar. ¿Seguimos siendo un país rentista con toda la mentalidad que ello crea o decidimos ser un país productor? ¿Vivimos otro siglo del petróleo o erigimos una economía de raíz productiva? Es el dilema de mayor transcendencia que nos plantea el nuevo siglo. Para resolverlo, necesitamos renovar, hasta el alzamiento contra lo viejo la filosofía y el estilo con los que siempre se ha planteado la cuestión del petróleo. Desde Alberto Adriani hasta Juan Pabló Pérez Alfonzo, para citar a los dos venezolanos que han abordado este tema con mayor solvencia, se ha considerado que el petróleo debe ser el motor que imparta el impulso dinámico a la economía. Fuente de recursos de inversión, vehículo de modernización económica, despensa para los bie-

13 "La crisis tiene su origen en la ruptura de la condición de equilibrio para un crecimiento armónico. Esta condición de equilibrio viene determinada por la necesidad de que el consumo y la inversión crezcan de manera simultánea en una cierta proporción” (Baptista, 2004, p. 57).
La razón rentista y sus teóricos: la economía política en el siglo petrolero venezolano Malfred Gerig 
Dossier América Latina: Venezuela nes de consumo, el petróleo tendría la responsabilidad de dispensarnos esos medios. (Rangel, 2004, p. 230)

El dilema planteado por Rangel se acrecentaría con el giro de los acontecimientos. Las exportaciones petroleras como porcentaje de las exportaciones totales ascendieron del 71,6 \% (su mínimo desde 1962) en 1998 hasta el 98,7\% en 2012 (su máximo histórico) (BM, 2020). Venezuela entró en una belle époque del siglo petrolero venezolano en la que se propiciaron todos y cada uno de los desequilibrios macroeconómicos precedentes ${ }^{14}$, que se extendió desde 2004 a 2012. A partir de allí, el país ingresó en una dinámica que transcurrió de la crisis al colapso, del colapso a la extinción.

Paradójicamente, el colapso no ha llevado a zanjar el dilema entre rentistas o productores, ya que, como sostenía el propio Domingo Alberto Rangel, "ninguna sociedad en la historia, de las tantas que gozaron una renta o vivieron un sistema rentístico transformó su naturaleza para evitarse el eventual embarrancamiento (...) Las sociedades como la gente se enmiendan cuando la catástrofe que despreciaron les cae encima hasta aplastarlas" (2004, p. 232). Sigue siendo crucial pensar las cosas de petróleo de nueva cuenta, pese a que los logros del siglo petrolero venezolano se escurran en nuestras manos. Pensar al petróleo ya no bajo la matriz de la razón rentista, sino en la matriz de un pensamiento que supere al tiempo que conserva. El dilema nacional está planteado entre un nacionalismo tercero, capaz de hacer síntesis entre desarrollismo y dependentismo, o el fin de Venezuela como país petrolero. Para hacer frente a la razón rentista es necesario conservar aquello que Gramsci (1984, p. 27) denominó “espíritu estatal”, para así, con las lecciones de la tradición -tanto la que se ocupó de legitimar el ingreso rentista como la que procuró la recta caracterización- hacer frente a un futuro en el cual Venezuela puede ser un país petrolero, pero de ningún modo podrá seguir siendo un país rentista.

14 La dependencia de la economía venezolana del sector petrolero se agravó, se hicieron avances en la recaudación fiscal, pero en ningún caso con los niveles de profundidad necesarios. La sobrevaluación del bolívar y el flujo de divisas favorecieron el empoderamiento de la burguesía comercial en detrimento de la muy mermada burguesía productiva. La caída de la productividad continuó su marcado declive pese a las inyecciones de capital fijo en algunos sectores, la crisis de inversión privada llegó a su cenit, igualmente, la dependencia de las importaciones como forma de tapar los huecos productivos se aceleró. Pero sobre todo no hubo ningún intento de resolver los problemas de reproducción ampliada: se importaron medios de consumo y cada vez la capitalización de la renta era menor. 


\section{Referencias bibliográficas}

Arrighi, G. (1999). El largo siglo XX: dinero y poder en los orígenes de nuestra época. Madrid: Akal.

Banco Mundial (BM) (2020). Datos de libre acceso del Banco Mundial. Recuperado de https://datos.bancomundial.org/

Baptista, A. (2004). El relevo del capitalismo rentístico: hacia un nuevo balance de poder. Caracas: Fundación Polar.

Baptista, A. (2010). Teoría económica del capitalismo rentístico (2da ed.). Caracas: Banco Central de Venezuela.

Baptista, A. y Mommer B. (1992). El petróleo en el pensamiento económico venezolano: un ensayo. Caracas: Ediciones IESA.

Baptista, B. (2008). Itinerario por la economía política. Caracas: Ediciones IESA.

Crazut, R. J. (2010). El Banco Central de Venezuela: notas sobre su historia y evolución en sus 70 años de actividades (5ta ed.). Caracas: Banco Central de Venezuela.

Egaña, M. R. (2010). Venezuela y sus minas. Caracas: Banco Central de Venezuela.

Furtado, C. (1993). Notas sobre economía venezolana. En Los vientos del cambio (pp. 258278). México: Fondo de Cultura Económica.

Gerig, M. (2019). Un trazado de acontecimientos: la economía política de la desposesión, el ascenso de Asia oriental y la crisis de la geocultura liberal. RIPS: Revista de Investigaciones Políticas $y$ Sociológicas, vol. 18, no. 2, pp. 115-136. DOI: http://dx.doi.org/10.15304/rips.18.2.5387

Gramsci, A. (1984). Notas sobre Maquiavelo, sobre la política y sobre el estado moderno. Buenos Aires: Nueva Visión.

Gramsci, A. (2004). Análisis de situaciones correlaciones de fuerzas. En Antología (sel., trad. y ns. Manuel Sacristán) (pp. 409-422). Buenos Aires: Siglo XXI.

Lieuwen, E. (2016). El petróleo en Venezuela: una historia. Caracas: El Perro y la Rana.

Marx, K. (1975). El capital: Crítica a la economía política. Tomo I: El proceso de producción del capital. México: Siglo XXI, Vol. I y II.

Marx, K. (1985). El capital: crítica de la economía política. Tomo III: El proceso global de la producción capitalista. México: Siglo XXI, Vol. III.

Mendoza Potellá, C. (2011). De las concesiones a los contratos: visión retrospectiva de la política petrolera venezolana. Caracas: El Perro y la Rana.

Mommer, B. (2010). La cuestión petrolera (3era ed.). Caracas: Fondo Editorial Darío Ramírez/PDVSA.

Panitch L. y Gindin, S. (2015). La construcción del capitalismo global: la economía política del imperio estadounidense. Madrid: Akal.

Pérez Alfonzo, J. P. (1971). Petróleo y dependencia. Caracas: Síntesis Dos Mil.

Pérez Alfonzo, J. P. (2011). Hundiéndonos en el excremento del diablo. Caracas: Banco Central de Venezuela.

Rangel, D. A. (2004). Venezuela en tres siglos (2da ed.). Caracas: Mérida Editores.

Rangel, D. A. (2006). Los Andinos en el poder: balance de historia contemporánea 1899-1945 (4ta ed.). Mérida: Mérida Editores.
La razón rentista y sus teóricos: la economía política en el siglo petrolero venezolano Malfred Gerig 
Dossier Rodríguez, L. C. (2015). Gómez: agricultura, petróleo y dependencia (2da ed.). Caracas: América Latina: Centro Nacional de Historia.

Venezuela

Schumpeter, J. (1968). Capitalismo, socialismo y democracia. Barcelona: Orbis.

Tinker Salas, M. (2013). Una herencia que perdura: petróleo, cultura y sociedad en Venezuela. Caracas: Galac.

Uslar Pietri, A. (2006). Pasión de Venezuela. Suplemento de la Revista BCV, XX (2), Caracas.

Wallerstein, I. (2004). El ascenso y futura decadencia del sistema-mundo capitalista: conceptos para un análisis comparativo. En Capitalismo histórico y movimientos antisistémicos (pp. 85114). Madrid: Akal.

Wallerstein, I. (2014). El moderno sistema mundial, Vol. IV: El triunfo del liberalismo centrista, 1789-1914. México: Siglo XXI.

Yergin, D. (1992). La historia del petróleo. Barcelona: Plaza y Janes. 